\title{
Review: black people are more likely than white people to be detained in psychiatric wards in the United Kingdom
}

Bhui K, Stansfeld S, Hull S, Priebe S et al. Ethnic variations in pathways to and use of specialist mental health services in the UK. Systematic review. Brit J Psychiatry 2003 Feb, 182:105-16.

\section{QUESTION: Are there ethnic differences in pathways to specialist mental health care; continuing contact with mental health services; use of in-patient services, and compulsory admissions in the United Kingdom?}

\section{Design}

Systematic review with narrative synthesis and metaanalysis.

Source of funding:

London National

Health Service,

Research and

Development.

For correspondence:

K Bhui, Department of

Psychiatry, Barts and

the London, Queen

Mary's School of

Medicine and

Dentistry, UK.

k.s.bhui@qmul.ac.uk

\section{Data sources}

The authors searched ASSIA, CINAHL, the Cochrane Trials Register, Embase, HealthSTAR, Medline, PsycLit, the Science Citation Index, SIGLE and personal files for studies published in English between January 1983 and October 2000. The authors sought grey literature and other unpublished literature from UK Health Authorities. Experts in the field were asked to identify omissions from a list of identified articles.

\section{COMMENTARY}

This review collates research from the United Kingdom on pathways to care and the prevalence of mental health service use by different ethnic groups. Variability in study design, definitions of ethnic groups, and outcome measures made it difficult to synthesise many of the findings quantitatively, but the meta-analysis suggests that black people are significantly more likely than white people to be compulsorily detained.

Discrimination may be one cause of variations in service use. The Police are more likely to make emergency referrals to psychiatric services for black people. Some suggest that this reflects prejudice against black people and less tolerance of their behaviour in public. ${ }^{1}$ This in turn affects future use of services. A circle can develop of greater and greater dislike of psychiatric care leading to repeated compulsory admissions. Asian patients may be discriminated against by being stereotyped as somatizers by primary care practitioners.

Factors such as social support are also important. For example, some patients may use inpatient services less because of greater family involvement at times of crisis. More research is needed, particularly into what happens in the community, in primary care, and at the primary-secondary care interface, using careful descriptions of ethnic group, pathways to care, and outcomes.

UK primary care organisations have recognised problems of access for people of different ethnic groups, refugees, and asylum seekers. ${ }^{2}$ They have responded through initiatives to employ doctors, nurses, other health professionals, and interpreters to target those with greater needs who are less likely to access services. ${ }^{2}$ These initiatives have not been evaluated. This is a complex issue involving interactions between supply and demand which depend on the preferences of patients and practitioners. ${ }^{3}$

For many physical problems, tackling inequality involves providing equal access to services, but mental health services may actually aggravate a person's problems as well as helping. ${ }^{1}$ Overcrowding, lack of therapeutic input, lack of recreation, physical danger and coercion persist in UK psychiatric wards, even those in district general hospitals alongside wards for physical illness. ${ }^{1}$ Tackling inequalities in access to services is clearly important, but reviewing the nature and quality of the services offered is also a priority. Professor Tony Kendrick, MD FRCGP FRCPsych Primary Medical Care University of Southampton, United Kingdom

1 Rogers A. Inequalities created by service provision. In Pilgrim D, Rogers A. Mental Health and Inequality. Palgrave Publishers, 2002

Smith K, Wilkin D. Primary care development. In Wilkin D, Coleman A, Dowling B, Smith K (eds). The National Tracker Survey of Primary Care Groups and Trusts 2001/2002: Taking Responsibility. Manchester: University of Manchester, 2002.

Goddard M, Smith P.Equity of access to health care services: theory and evidence from the UK. Soc Sci Med 2001; 53:1149-62.

\section{Study selection}

Prospective, retrospective or cross sectional studies with quantitative data were eligible if they compared access to or use of primary or secondary mental health services by more than one ethnic group in the United Kingdom. Adults with psychotic and non-psychotic mental disorders were eligible. Specialist services for children, adolescents, and older people were excluded, as were forensic services and services for psychosexual disorders or substance abuse.

\section{Data extraction}

Two reviewers assessed papers independently for relevance and extracted data. Disagreements were resolved by a third reviewer. Meta-analyses were generally inappropriate because research design, quality, ethnic group definitions and outcome measures varied between studies. After performing tests for heterogeneity, the authors combined data on the proportions of black and white patients compulsorily admitted to psychiatric wards.

\section{Main results}

The authors included 38 papers. Most compared black and white people. Most reported cross sectional studies, without distinguishing incident versus prevalent cases. 34 studies were based on data from medical records, 15 included interview data and 10 used self complete surveys. The majority of studies were subject to recall bias and information bias. Most did not differentiate between subgroups (such as subgroups with the "white" ethnic group or differences within "Asian" populations).

Despite these methodological problems, the findings suggest that black people are more likely than white people to be voluntary or compulsory inpatients in psychiatric wards (pooled odds ratio for compulsory admission of black versus white patients $4.31,95 \%$ CI 3.33 to 5.58). Black patients had more varied and complex pathways to specialist care. Black people were also more likely to enter care through "crisis routes" such as police intervention. There was some ethnic variation in primary care assessments, which may explain some differences in the use of specialist services. The authors note that qualitative studies that might help to explain the quantitative findings were excluded from the review.

\section{Conclusions}

Voluntary and compulsory admissions to specialist mental health services vary between ethnic groups. There is some evidence that pathways to specialist care also vary. 